in vivo $34: 1857-1862(2020)$

doi:10.21873/invivo.11980

\title{
Osteogenic Evaluation of Hydroxyapatite Scaffold Loaded With Dexamethasone in Femoral Drill Holes
}

\author{
MIN-HO PARK ${ }^{1 *}$, SEOK JIN JANG ${ }^{2 *}$ and SEOK HWA CHOI ${ }^{1}$ \\ ${ }^{1}$ Department of Veterinary Surgery, College of Veterinary Medicine, \\ Chungbuk National University, Cheongju, Republic of Korea; \\ ${ }^{2}$ Onnuri Animal Medical Center, Cheongju, Republic of Korea
}

\begin{abstract}
Background/Aim: Many cases of bone damage are due to trauma and metabolic diseases. This study aimed to evaluate bone regeneration into a porous hydroxyapatite (HA) scaffold using dexamethasone (DM)-loaded polymeric microspheres. Materials and Methods: Four adult dogs were used to evaluate the in vivo performance of DM-loaded microspheres immobilized on the surfaces of porous HA scaffolds. Two 5-mm drill holes were created in both the left and right femurs of each dog. The experimental groups included a control group (drill holes filled with HA scaffold alone), a DM 20 group (holes filled with DM-loaded HA scaffold with $20 \mathrm{mg}$ DM per scaffold), and a DM 100 group (hole filled with DM-loaded HA scaffold with $100 \mathrm{mg}$ DM per scaffold). Resulting bone volume percentages and bone mineral densities were calculated by examing microcomputed tomographic (CT) images. Results: The DMloaded HA scaffold groups showed a gradual periosteal reaction two weeks after insertion of the HA scaffold into the femoral drill holes. Four weeks after HA scaffold insertion, the periosteal reaction in the femoral drill holes became denser. Eight weeks after insertion of DM-loaded HA scaffolds, clear images of the scaffold were observed in micro-CT images of the femoral drill hole. The DM 100 group had better bone healing tendencies (bone mineral density, bone mass, trabecular volume, bone surface, and trabecular thickness) than the DM 20 group. Conclusion: DM-loaded HA scaffolds are suitable platforms for
\end{abstract}

This article is freely accessible online.

*These Authors contributed equally in this study.

Correspondence to: Seok Hwa Choi, DVM, Ph.D., College of Veterinary Medicine, Chungbuk National University, Chungdae-ro 1, Seowon-Gu, Cheongju, Chungbuk, 28644, Republic of Korea. Tel: +82 432613144, Fax: +82 432613224, e-mail: shchoi@cbnu.ac.kr

Key Words: Dexamethasone, drill bit, hydroxyapatite, osteogenesis, scaffold. distributing bioactive molecules during osteogenesis in femoral drill holes.

In recent years, the incidence of bone tumors tends to markedly increase, especially in populations where aging is associated with increased obesity and low levels of physical activity. Management of segmental defects in long bones remains a difficult clinical problem. In particular, critical bone defects are more serious. For the reconstruction of such defects, autografts, allografts, or xenografts have been used (1). Although autografts are good for inducing osteoinduction and osteoconduction, such grafts are limited to filling small defects because they are limited by the amount of tissue that can be obtained. Artificial synthetic bone grafts have been regarded as a potential alternative to the conventional use of bone grafts because of their unlimited supply and the absence of disease transmission. Synthetic hydroxyapatite (HA) is commonly used as an artificial bone graft substance due to its inherent bioactive properties that support osteoconduction when used for bone regeneration. Moreover, HA has an excellent biocompatibility because its composition resembles the apatite occurring in natural bone (2).

Although pure HA is biologically active, it is difficult to incorporate therapeutics into HA without destroying the biological functions of the HA surface. Several approaches to incorporating therapeutics into porous HA scaffolds have been attemped, including immersing porous HA scaffolds in therapeutic solution or coating them with polymer solutions that contain therapeutic molecules (3). Poly(lactic-coglycolic acid) (PLGA) is a synthetic polymer used in tissue engineering and drug delivery. PLGA is a biodegradable polymer with excellent processibility for the generation a flexible structure and its degradation rate can be controlled (4). Traditionally considered a superior controlled-release carrier, PLGA microspheres have ideal loading efficiency and can optimize drug dosage.

Dexamethasone (DM) induces osteoblastic differentiation in vitro by increasing alkaline phosphatase activity, osteocalcin expression, and bone sialoprotein levels (5). DM 
is used for the prevention of inflammation in orthopedic surgery and has a well-known effect on bone density. Shortterm DM treatment has been shown to change osteocyte and osteoblast activities (6). It can also suppress the bonedegrading capacity of osteoclasts (7) and increase bone resorption (6). In this study, we envisioned the use of a HA scaffold containing PLGA microspheres loaded with DM as bone substitutes to induce bone regeneration. The DM was used as a model bioactive molecule. The study evaluated bone regeneration associated with DM-loaded PLGA microspheres immobiliaed on HA scaffold surfaces placed in cavities created in canine femurs.

\section{Materials and Methods}

Preparation of porous HA scaffolds. HA scaffolds were fabricated using the polyurethane sponge replication method (8). A polyurethane sponge was coated with HA powders (OssGen Co., Daegu, Republic of Korea) in a distilled water-based slurry. Coated sponges were dried overnight at room temperature before sintering in a high temperature furnace at $1,230^{\circ} \mathrm{C}$ for $3 \mathrm{~h}$. The dimensions of the fabricated HA scaffold were $5 \mathrm{~mm}$ in diameter and $5 \mathrm{~mm}$ in length.

DM-loaded PLGA microspheres. One gram of PLGA and $100 \mathrm{mg}$ of DM (Sigma-Aldrich, St. Louis, MO, USA) were dissolved in 10 $\mathrm{ml}$ of dichloromethane/ethanol $(9: 1 \mathrm{v} / \mathrm{v})$ and added to $100 \mathrm{ml}$ of a $0.2 \%$ aqueous solution of polyvinyl alcohol, followed by homogenizing (PowerGen 500, Fisher Scientific, NY, USA) at $4480 \times \mathrm{g} / \mathrm{min}$ for $3 \mathrm{~min}$. The emulsified solution was then poured into a beaker containing $300 \mathrm{ml}$ of $0.5 \%$ polyvinyl alcohol and stirred under a hood with a magnetic stirrer for $4 \mathrm{~h}$ to evaporate the solvent. The hardened microspheres were collected by centrifugation at $1,008 \times \mathrm{g} / \mathrm{min}$ for $3 \mathrm{~min}$, washed with distilled water water, and lyophilized using a freeze dryer. The resulting samples were stored in a desiccator under vacuum at $-20^{\circ} \mathrm{C}$. The molar ratio of the lactide/glycolide of the PLGA polymer (Boehringer Ingelheim, Ingelheim, Germany) was 75:25.

Radiographic and computed tomographic (CT) image observations. Structural features of the DM-loaded HA scaffold were observed using a Micromaster stereoscope (Fisher Scientific, NY, USA) and a scanning electron microscope (EVO 40, Carl Zeiss, Wilmington, DE, USA). Examination of clinical signs and radiographic and micro-CT images was performed to evaluate the serial healing process during the experimental period. Plain radiographs were obtained at 0,2 , and 4 weeks after insertion of the HA scaffolds to evaluate the correct positioning of the scaffolds. The dogs' exercise was restricted for two weeks after treatment and the healing process was assessed by examining both radiographic and micro-CT images.

To evaluate new bone formation, femoral samples were scanned by using a micro-CT apparatus (Bruker MicroCT, Kontich, Belgium) at $100 \mathrm{kV}$ source voltage and $100 \mu \mathrm{A}$ source current with no filter and at a spatial resolution of $8.77 \mu \mathrm{m}$. Reconstructions were performed using the NRecon software (Skyscan, Seoul, Republic of Korea). The bone density of cortical bones was measured by determing Hounsfield units (HU). The percent bone volume and bone mineral density (BMD) values were calculated from micro-CT image information.

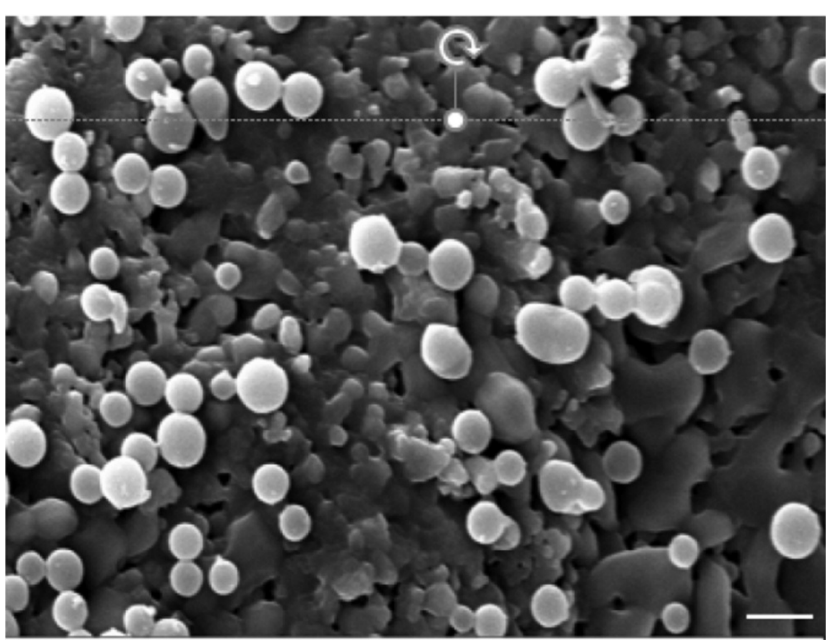

Figure 1. Scanning electron micrograph of dexamethasone (DM)-loaded poly(lactic-co-glycolic acid) (PLGA) microspheres immobilized onto a porous HA scaffold surface. Scaffold pore sizes ranged from $230 \mu \mathrm{m}$ to $470 \mu \mathrm{m}$ and porosity was $91.6 \pm 1.2 \%$. Bar scale $=2 \mu \mathrm{m}$.

In vivo study. The two-year-old male beagle experimental animals weighed $7-9 \mathrm{~kg}$. In each of four dogs, we created two $5-\mathrm{mm}$ drill holes in both the left and right femurs under general anesthesia. The experimental group of 16 drill holes was divided into a control group (drill holes filled with HA scaffold alone, $n=6$ ), a DM 20 group (holes filled with DM-loaded HA scaffold with $20 \mathrm{mg}$ DM per scaffold, n=5), and a DM 100 group (holes filled with DMloaded HA scaffold with $100 \mathrm{mg}$ DM per scaffold, $n=5)$. Femoral drill holes were evenly distributed among the four dogs, using a Lartin square design. This experimental procedure was approved by the Institutional Animal Care and Use Committee of Chungbuk National University (Approval CA-18-23), Republic of Korea. A course of antibiotics and analgesics were administered for 7 days after hole drilling and the wound was cleaned daily by an antiseptic solution. Dogs were clinically observed daily during the experimental period to record postoperative complications.

Statistical analysis. Statistical analyses were performed using the SPSS software version 19.0.1.1. (IBM SPSS Statistics for Windows, Version 19.0; IBM Corp., Armonk, NY, USA). Data are presented as mean \pm standard deviation (SD) values. Normality and homogeneity of the data were confirmed before performing an analysis of variance (ANOVA). Differences among the experimental groups were assessed by performing one-way ANOVA followed by Duncan's multiple range tests. Null hypotheses of no significant difference were rejected if the obtained $p$-values were less than 0.05 .

\section{Results}

Characterization of DM-loaded HA scaffolds. The HA scaffold had a very high porosity with good interconnections among the scaffold's pores. Open channels were arranged with isotropic geometry and exhibited a rounded-edge strut morphology. Pore sizes ranged from $230 \mu \mathrm{m}$ to $470 \mu \mathrm{m}$ and 


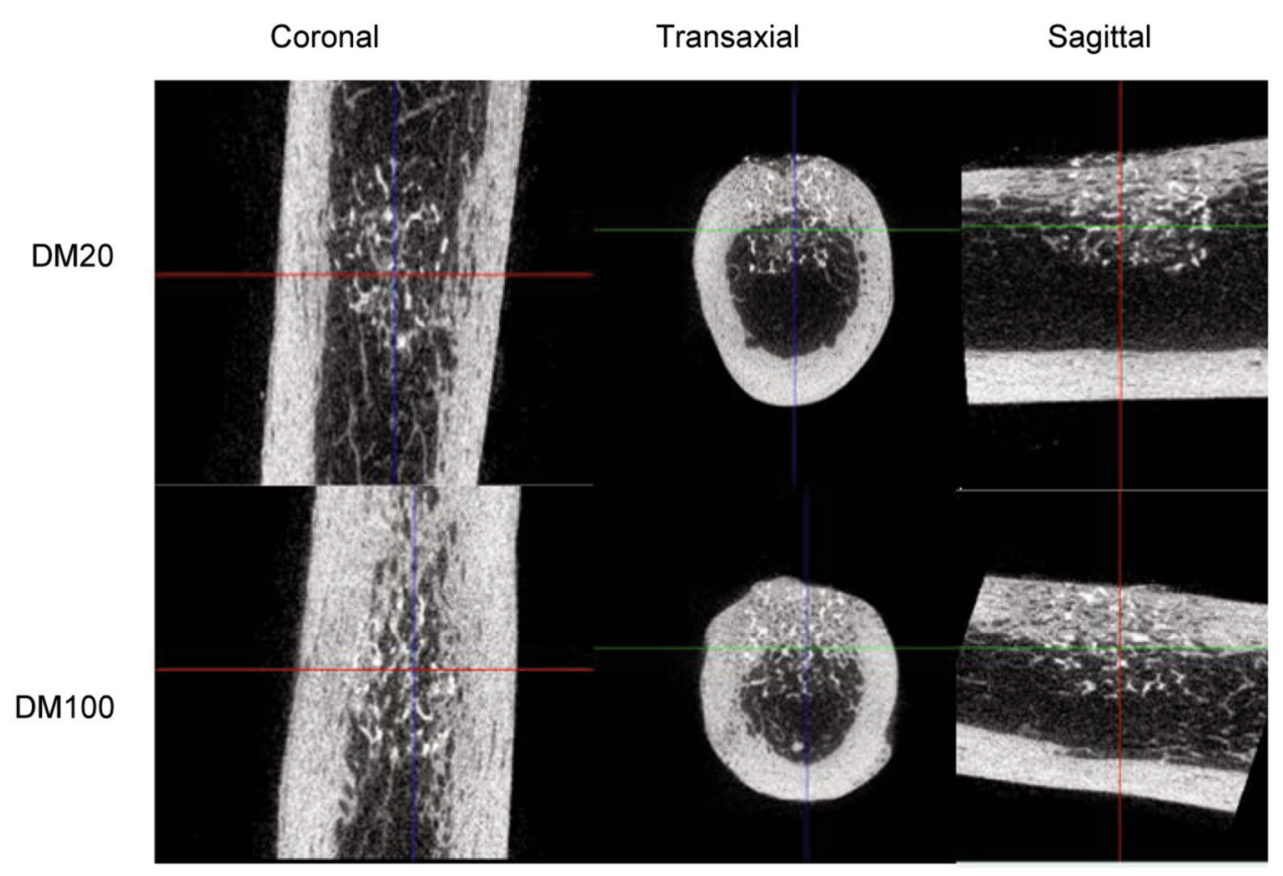

Figure 2. Micro-computed tomography (CT) images obtained 8 weeks after insertion of dexamethasone (DM)-loaded HA scaffold into holes drilled in canine femurs.

mean porosity was $91.6 \pm 1.2 \%$. In general, the HA scaffold structure resembled the human bone structure. DM agents were shown to adhere efficiently to the microspheres containing a porous HA scaffold (Figure 1).

Radiographic and micro-CT observations. Beagle dogs showed stable vital signs for 8 weeks after insertion of the DM-loaded HA scaffolds, and the surgical sites of all femurs healed well. Eight weeks after the insertion of the DM-loaded HA scaffold, mico-CT imaging revealed that the scaffold was securely attached to the femoral drill hole (Figure 2). The mean HU values of the DM-loaded HA scaffold groups indicated higher bone densities than in the control group (Table I). Moreover, the BMD values and percent bone volumes in the DM 100 group were higher than those of the DM 20 group (Table II). In the DM-loaded groups, periosteal reactions in the femoral drill holes gradually increased from 2 weeks after HA scaffold insertion. Four weeks after the insertion of DM-loaded HA scaffolds, densities of the periosteal reactions in the femoral drill holes had increased. Eight weeks after the insertion of HA scaffolds, micro-CT images showed that the drill holes in the DM-loaded groups had mineralized, and new bone formation was visible. The DM-loaded HA scaffold groups had improved both the quantity and quality of new bone formation compared to that of the control group (Table II). Eight weeks after the insertion of DM-loaded HA scaffolds, clear images of the inserted HA
Table I. Comparison of micro-computed tomography (CT) results obtained before and 8 weeks after insertion of DM-loaded HA scaffolds.

\begin{tabular}{lcc}
\hline Group & \multicolumn{2}{c}{ Weeks after HA scaffolds insertion } \\
\cline { 2 - 3 } & 0 Weeks & 8 Weeks \\
\hline Control & $279.88 \pm 20.12$ & $977.15 \pm 126.29$ \\
DM 20 & $310.78 \pm 17.36$ & $1036.07 \pm 12.12$ \\
DM 100 & $371.67 \pm 55.68 *$ & $1070.20 \pm 34.93 *$ \\
\hline
\end{tabular}

Data are expressed as mean \pm SD Hounsfield units $(n=5)$. *Significant difference from the control group at $p<0.05$. HA: Hydroxyapatite; DM: dexamethasone.

scaffolds were visible on micro-CT scans of the canine femurs (Figure 2). The BMD, bone volume, bone surface, and cortical bone thickness in the DM 100 group were superior to those in the DM 20 group. All DM-loaded HA scaffold groups showed improved bone healing over that of the control group.

\section{Discussion}

HA is widely used in orthopedic and dental surgery as a bone substitue because its constituents are similar to those of natural bone apatite $(9,10)$. HA has an interconnected porous and isotropic structure, and many studies have been 
Table II. Bone parameters measured by micro-computed tomography (CT) at 8 weeks after insertion of DM-loaded HA scaffolds.

\begin{tabular}{lcccccc}
\hline Group & \multicolumn{2}{c}{ BMD $\left(\mathrm{g} / \mathrm{cm}^{3}\right)$} & & $\begin{array}{c}\text { BV/TV } \\
(\%)\end{array}$ & $\begin{array}{c}\text { BS/BV } \\
\left(\mathrm{mm}^{-1}\right)\end{array}$ & $\begin{array}{c}\mathrm{BS} / \mathrm{TV} \\
\left(\mathrm{mm}^{-1}\right)\end{array}$ \\
\cline { 2 - 6 } & Trabecular & Cortical & & & & $\begin{array}{c}\mathrm{Tb} . \mathrm{Th} \\
(\mathrm{mm})\end{array}$ \\
\hline Control & $0.48 \pm 0.06$ & $0.97 \pm 0.07$ & $61.62 \pm 2.50$ & $13.94 \pm 1.53$ & $8.57 \pm 0.59$ & $0.23 \pm 0.02$ \\
DM 20 & $0.53 \pm 0.17$ & $1.17 \pm 0.14$ & $62.66 \pm 5.46$ & $13.98 \pm 1.17$ & $8.79 \pm 1.50$ & $0.25 \pm 0.01$ \\
DM 100 & $0.60 \pm 0.19^{*}$ & $1.02 \pm 0.15$ & $65.03 \pm 9.87$ & $14.28 \pm 1.86$ & $9.20 \pm 0.20$ & $0.25 \pm 0.03$ \\
\hline
\end{tabular}

Data are expressed as mean $\pm \mathrm{SD}(\mathrm{n}=5)$. *Significant difference from the control group at $p<0.05$. BMD: Bone mineral density, BV: bone volume, TV: trabecular volume, BS: bone surface, Tb.Th: trabecular thickness.

attempted to integrate therapeutic agents into the HA streucture $(8,11)$. PLGA microsphere materials are used in a wide range of applications due to their biocompatibility and biodegradability. Moreover, PLGA microspheres can be retained in cytoplasm or extracellular spaces to slowly release an encapsulated drug via hydrolysis and drug diffusion from the microspheres. Such slow intracellular release can result in sustained intracellular drug delivery (12). These features should contribute to a highly efficient targeted delivery of drugs eluted from a HA scaffold with embedded microspheres. Therefore, we believe that placing PLGA microspheres within a porous HA scaffold will provide an effective platform for delivering bioactive molecules for bone damage or disease treatment. In this study, we developed a porous HA scaffold containing DMloaded PLGA microspheres for use as a drug-delivery platform for bone regeneration. DM was used as the model bioactive molecule and PLGA microspheres as the carrier.

$\mathrm{DM}$ is a synthetic corticosteroid, widely used to produce antiemetic, analgesics, anti-inflammatory and immunosuppressive effects in dental and orthopedic surgery (13), thyroidectomy $(14,15)$, and hysterectomy $(16)$. It has been suggested that the vomiting prevention effect may be due to suppression of prostaglandins, prevention of serotonin release in the gut, reduced levels of neuronal 5hydroxytryptophan, or the release of endorphin (17). It has been reported that preoperative DM reduces postoperative nausea, sustains the inhibitory antiemetic effect and reduces dynamic pain at $24 \mathrm{~h}$ after a total hip replacement (18). In addition, DM is widely used in clinical practice before and after surgery for anti-inflammatory purposes. The antiinflammatory effect of DM is approximately 30 times more potent than that of cortisol. Previous clinical studies have applied a range of DM dosages in humans $(18,19)$.

Some researchers have reported that DM-loaded HA has an effect on bone regeneration in vitro (5), while the present study was conducted to confirm that it is also effective in vivo. Some researchers have reported that the effective DM concentration to induce osteogenic differentiation of bone marrow stromal cells is in the range of $40-400 \mathrm{ng} / \mathrm{ml}$ and that DM has toxic effects at 4,000 ng/ml (20). However, DM has potential adverse effects, especially when administered systemically at high doses over a long period of time $(21,22)$. Moreover, some researchers have reported potential side effects from a single dose of DM $(23,24)$. In this study, we thought to minimize these potential adverse effects by localizing the DM in the surgery site.

This study evaluated the regenerative response of bone to a DM-loaded HA scaffolds acting as antiinflammatory carriers with a sustained release of DM. Based on previous studies $(11,25)$ we hypothesized that the DM-loaded HA scaffold might promote bone regeneration in holes drilled into canine femurs. In this study, the DM-loaded HA scaffold was shown to increase osteogenesis in drill holes in canine femurs and to be effective as an anti-inflammatory carrier.. The femurs with DM-loaded HA scaffolds had lower inflammatory responses than thoses of femurs with HA scaffolds alone. In the present study, bone regeneration was proportional to the dose of DM. Our results supported those of an earlier report showing that DM has an antiinflammatory effect early in the postoperative period (25). The anti-inflammatory effect is complex but functions mainly through the suppression of inflammatory cells and expression of inflammatory mediators (22).

For effective bone regeneration in orthopedic surgery, the optimal concentration of DM in a DM-loaded HA scaffold must be determined and further study is needed to determine that concentration. DM has a significant effect on the skeleton, especially when treated over a long period of time. Moreover, high-dose DM therapy is commonly associated with bone loss and can produce one of the most crippling forms of osteoporosis (7). However, in this study, osteoporosis was not detected in the CT images obtained during the experimental period. In addition, we did not observe any DM-related clinical side effects in the treated dogs. We speculate that these positive results are due to the short-term application of low-dose DM.

Our results are consistent with those reported by Kim et al. (11), a study that developed a microsphere system for continuous delivery of DM, a corticosteroid antiinflammatory drug. In our study, the selected DM dose 
minimized inflammation in the femoral drill holes, but the optimal DM dosage requires further study. The DM-loaded HA scaffold provided in this study was an effective platform for delivering bioactive molecules to femoral drill hole treatment sites. The platform was able to induce osteogenesis in vivo and we believe this type of platform can be useful when applied as an implantable artificial bone graft to improve osteogenesis in vitro.

\section{Conflicts of Interest}

The Authors declare that they have no competing interests regarding this study.

\section{Authors' Contributions}

This study was designed by Park and Jang. Jang, Jang and Choi analyzed the CT image and data collection. All Authors read and approved the final manuscript.

\section{Acknowledgements}

This research was supported by the Basic Science Research Program provided through the National Research Foundation of Korea (NRF) and funded by the Ministry of Education (2018R1D1A1A09083556).

\section{References}

1 Jang SJ and Choi SH: Evaluation of goose-beak bone particles for dentoalveolar reconstruction in dogs. In Vivo 34: 609-614, 2020. PMID: 32111760. DOI: 10.21873/invivo.11814

2 Liu DM: Fabrication and characterization of porous hydroxyapatite granules. Biomaterials 17: 1955-1957, 1996. PMID: 8894087. DOI: 10.1016/0142-9612(95)00301-0

3 Kim HW, Knowles JC and Kim HE: Hydroxyapatite/poly( $\varepsilon-$ caprolactone) composite coatings on hydroxyapatite porous bone scaffold for drug delivery. Biomaterials 25: 1279-1287, 2004. PMID: 14643602. DOI: 10.1016/j.biomaterials.2003.07.003

4 Giteau A, Venier-Julienne MC, Aubet-Pouessel A and Benoit JP: How to achieve sustain and complete protein release from PLGAbased microparticles? Int J Pharm 350: 14-26, 2008. PMID: 18162341. DOI: 10.1016/j.ijpharm.2007.11.012

5 Cheng SL, Yang JW, Rifas L, Zhang SF and Avioli LV: Differentiation of human bone marrow osteogenic stromal cells in vitro: induction of the osteoblast phenotype by dexamethasone. Endocrinol 134: 277-286, 1994. PMID: 8275945. DOI: 10.1210/endo.134.1.8275945

6 Brabnikova Maresova K, Pavelka K and Stepan JJ: Acute effects of glucocorticoids on serum markers of osteoclasts, osteoblasts, and osteocytes. Calci Tissue Int 92: 354-361, 2013. PMID: 23247536. DOI: $10.1007 / \mathrm{s} 00223-012-9684-4$

7 Kim HJ, Zhao H, Kitaura H, Bhattacharyya S, Brewer JA, Muglia LJ, Ross FP and Teitelbaum SL: Glucocorticoids suppress bone formation via the osteoclast. J Clin Invest 116: 2152-2160, 2006. PMID: 16878176. DOI: 10.1172/JCI28084

8 Son JS, Appleford M, Ong JL, Wenke JC, Kim JM, Choi SH and Oh DS: Porous hydroxyapatite scaffold with three-dimensional localized drug delivery system using biodegradable microspheres. J Control Release 153: 133-140, 2011. PMID: 21420453. DOI: $10.1016 /$ j.jconrel.2011.03.010

9 Kim JM, Han TS, Kim MH, Oh DS, Kang SS, Kim G, Kwon TY, Kim KH, Lee KB, Son JS and Choi SH: Osteogenic evaluation of calcium phosphate scaffold with drug-loaded poly (lactic-co-glycolic acid) microspheres in beagle dogs. Tissue Eng Regen Med 9: 175183, 2012. PMID: 26811110. DOI: 10.1007/s13770-012-0175-5

10 Jang SJ, Kim SE, Han TS, Son JS, Kang SS and Choi SH: Bone regeneration of hydroxyapatite with granular form or porous scaffold in canine alveolar sockets. In Vivo 31: 335-341, 2017. PMID: 28438860. DOI: 10.21873/invivo.11064

11 Kim JM and Choi SH: Osteogenic evaluation of goose-beak bones according to processing temperature. In Vivo 33: 419-424, 2019. PMID: 30804120. DOI: 10.21873/invivo.11489

12 Nakano K, Egashira K, Masuda S, Funakoshi K, Zhao G, Kimura S, Matoba T, Sueishi K, Endo Y, Kawashima Y, Hara K, Tsujimoto H, Tominaga R and Sunagawa K: Formulation of nanoparticleeluting stents by a cationic electrodeposition coating technology: efficient nano-drug delivery via bioabsorbable polymeric nanoparticle-eluting stents in porcine coronary arteries. JACC Cardiovasc Interv 2: 277-283, 2009. PMID: 19463437. DOI: 10.1016/j.jcin.2008.08.023

13 Baxendale BR, Vater $\mathrm{M}$ and Lavery KM: Dexamethasone reduces pain and swelling following extraction of third molar teeth. Anaesthesia 48: 961-964, 1993. PMID: 24843434. DOI: 10.1111/j.1365-2044.1993.tb07474.x

14 Worni M, Schudel HH, Seifert E, Inglin R, Hagemann M, Vorburger SA and Candinas D: Randomized controlled trial on single dose steroid before thyroidectomy for benign disease to improve postoperative nausea, pain and vocal function. Ann Surg 248: 1060-1066, 2008. PMID: 19092351. DOI: 10.1097/ SLA.0b013e31818c709a

15 Feroci F, Rettori M, Borrelli A, Lenzi E, Ottaviano A and Scatizzi M: Dexamethasone prophylaxis before thyroidectomy to reduce postoperative nausea, pain and vocal dysfunction: a randomized clinical controlled trial. Head Neck 33: 840-866, 2011. PMID: 25855820. DOI: 10.1002/hed.21543

16 Jokela RM, Ahonen JV, Tallgren MK, Marjakangas PC and Korttila KT: The effective analgesic dose of dexamethasone after laparoscopic hysterectomy. Anesth Analg 109: 607-615, 2009. PMID: 19608838. DOI: 10.1213/ane.0b013e3181ac0f5c

17 Henzi I, Walder B and Tramer M: Dexamethasone for the prevention of postoperative nausea and vomiting: a quantitative systematic review. Anesth Analg 90: 186-194, 2000. PMID: 10625002. DOI: 10.1097/00000539-200001000-00038

18 Kardash KJ, Sarrazin F, Tessler MJ and Velly AM: Single-dose dexamethasone reduces dynamic pain after total hip arthroplasty. Anesth Analg 106: 1253-1257, 2008. PMID: 18349202. DOI: 10.1213/ANE.0b013e318164f319

19 Movafegh A, Soroush AR, Navi A, Sadeghi M, Esfehani F and Akbarian-Tefaghi N: The effect of intravenous administration of dexamethasone on postoperative pain, nausea and vomiting after intrathecal injection of meperidine. Anesth Analg 104: 987-999, 2007. PMID: 17377120. DOI: 10.1213/01 ane.0000257926.07491.55

$20 \mathrm{Kim} \mathrm{H}$, Kim HW and Suh H: Sustained release of ascorbate-2phosphate and dexamethasone from porous PLGA scaffolds for bone tissue engineering using mesenchymal stem cells. Biomaterials 24: 4671-4679, 2003. PMID: 12951010. DOI: 10.1016/s0142-9612(03)00358-2 
21 Porte RM, Huckle WR and Coldstein AS: Effect of dexamethasone withdrawal on osteoblastic differentiation of bone marrow stromal cells. J Cell Biochem 90: 13-22, 2003. PMID: 12938152. DOI: $10.1002 /$ jcb.10592

22 Schacke H, Docke WD and Asadullah K: Mechanisms involved in the side effects of glucocorticoids. Pharmacol Ther 96: 23-43, 2002. PMID: 12441176. DOI: 10.1016/s0163-7258(02)00297-8

23 Kakodkar PS: Routine use of dexamethasone for postoperative nausea and vomiting: the case for. Anaesthesia 68: 889-891, 2013. PMID: 23848292. DOI: 10.1111/anae.12308

24 Bartlett R: Routine use of dexamethasone for postoperative nausea and vomiting: the case against. Anaesth 68: 892-896, 2013. PMID: 23848377. DOI: 10.1111/anae.12309
25 Wang Q, Wang J, Lu Q, Detamore MS and Berkland C: Injectable PLGA based colloidal gels for zero-order dexamethasone release in cranial defects. Biomaterials 31: 49804986: 2010. PMID: 20303585. DOI: 10.1016/j.biomaterials. 2010.02 .052

Received March 25, 2020

Revised April 6, 2020

Accepted April 14, 2020 